\title{
Viabilidade técnica de produção e propriedades de painéis de partículas de casca de amendoim
}

\author{
Technical production viability and properties \\ of particleboard made with peanut husks
}

\author{
Mariana Pilar Gatani ${ }^{1}$, Juliano Fiorelli ${ }^{4}$, Juan Carlos Medina ${ }^{2}$, \\ Ricardo Arguello ${ }^{1}$, Agustin Ruiz ${ }^{2}$, \\ Maria Fátima do Nascimento ${ }^{3}$, Holmer Savastano Jr. ${ }^{4}$
}

\footnotetext{
${ }^{1}$ Centro Experimental de la Vivienda Económica (CEVE) - Consejo Nacional de Investigaciones Científicas y Tecnológicas (CONICET), Córdoba, Argentina. e-mail: marianagatani@ceve.org.ar

${ }^{2}$ Instituto de Tecnología de la Madera - Universidad Nacional de Santiago del Estero. Facultad de Ciencias Forestales, Córdoba, Argentina.

e- mail: jcmedina@unse.edu.ar

${ }^{3}$ Laboratório de Madeira e de Estruturas de Madeiras - EESC/USP, São Carlos, SP, Brasil. e-mail: fati@sc.usp.br

${ }^{4}$ Departamento de Engenharia de Biossistemas - FZEA/USP, Pirassununga, SP, Brasil. e-mail: julianofiorelli@usp.br ; holmersj@usp.br
}

\section{RESUMO}

No presente trabalho, considera-se o estudo do potencial de utilização de resíduos da casca de amendoim, com o intuito de agregar valor a este material, por meio da fabricação de painéis de partículas aglomeradas com resina ureia-formaldeído, prensados à temperatura de $100{ }^{\circ} \mathrm{C}$. A qualidade dos painéis foi avaliada com base nas prescrições do documento normativo ASTM D 1037:2006 por meio de ensaios físico-mecânicos de densidade, inchamento em espessura, absorção de água e flexão estática. Os painéis avaliados apresentaram densidade média variando de 0,69 a $0,83 \mathrm{~g} \mathrm{~cm}^{-3}$, MOR 4,37 a 5,34 MPa e MOE 590 a 700 MPa, para painéis com e sem tratamento preservativo. É possível afirmar, baseando-se no documento normativo ANSI A208.1:1993, que o material apresenta potencial para utilização em ambientes internos, como revestimento superficial de residências, construções agrícolas, setor moveleiro e decorativo. Recomenda-se a intervenção no processo de fabricação do painel, para melhorar as propriedades mecânicas e possibilitar o uso estrutural do material.

Palavras-chave: Resíduos agrícolas. painel de partículas. casca de amendoim.

\begin{abstract}
In this study, the potential use of residues of peanut husks was verified, in order to add value to these materials through the manufacture of panels of particleboards. For this procedure, it was used ureaformaldehyde resin and the panels were mechanical pressed at a temperature of $100{ }^{\circ} \mathrm{C}$. The quality of the panels was evaluated based on the prescriptions of the normative document ASTM D 1037:2006, through physical and mechanical tests of characteristics, such as: density, swelling in thickness, water absorption and static bending. The panels evaluated showed bulk density varying between 0,69 a $0,83 \mathrm{~g} \mathrm{~cm}^{-3}$, MOR and MOE between 4,37 - 5,34 MPa and 590 - $700 \mathrm{MPa}$ respectively, for panels with and without preservative treatment. It is possible said to claim, based in the normative document ANSI A208.1:1993, that the material presents a potential for use in internal areas as superficial covering of residential buildings, agricultural buildings, furniture and decorative sectors. Intervention in the board production process is recommended, in order to improve the mechanical properties of the material and enable its structural use.
\end{abstract}

Keywords: Agriculture waste, Particleboard, Peanut husks. 


\section{INTRODUÇÃO}

A tendência contemporânea tem sido caracterizada pela generalização do conceito de globalização econômica entre nações do mundo todo. Nela se experimenta a associação de países com interesses comuns em garantir a manutenção dos mercados e buscar sua expansão em um cenário fortemente marcado pela competitividade e pela necessidade de alcançar soluções inovadoras para os mais variados problemas. $\mathrm{O}$ Brasil tem buscado opções para aumentar a atividade econômica e uma das alternativas é o incentivo ao desenvolvimento de políticas no setor agrícola. A Argentina, um país com base econômica de produção agrária, tem a oportunidade de processar seus resíduos, agregando valor à produção primária. A busca por alternativas tecnológicas para o aproveitamento dos resíduos provenientes da industrialização dos grãos promove uma mudança no circuito de produção neste país.

A Comissão Mundial sobre Meio Ambiente e Desenvolvimento (CMMAD) define desenvolvimento sustentável como um processo de transformação no qual a exploração de recursos, o direcionamento de investimentos e a orientação do avanço tecnológico se harmonizam e reforçam o potencial presente e futuro, a fim de atender às necessidades da sociedade [1].

O setor agroindustrial depende direta ou indiretamente do ambiente como fonte de matérias-primas para o seu desenvolvimento, bem como da utilização de alguns locais para "área de despejo" de seus subprodutos e resíduos gerados durante os ciclos produtivos. A eliminação de resíduos, uma vez quantificados, controlados e tratados, torna-se facilmente passível de depuração pelo ambiente em determinado tempo. Caso contrário, esse processo pode levar milhares de anos ou, até mesmo, deixar de ocorrer, pela ausência de mecanismos específicos na natureza.

As indústrias de painéis de madeira reconstituída (partículas e fibras) na América Latina utilizam, preferencialmente, madeira de reflorestamento, provenientes de Pinus e de algumas espécies de Eucalyptus, o que determina, inclusive, a melhor qualidade do produto, tendo em vista o melhor controle de homogeneidade da matéria-prima. Porém, os materiais lignocelulósicos provenientes de resíduos agroindustriais vêm sendo utilizados com sucesso na fabricação de painéis como, por exemplo, o bagaço de cana-de-açúcar na confecção de painéis de partículas aglomeradas [2].

A reciclagem e a reutilização de materiais pós-consumo é um assunto que vem sendo frequentemente relatado em pesquisas científicas. Dentre os resíduos sólidos urbanos, o papel é um dos materiais que se encontram em maior volume nos lixões e aterros sanitários das cidades brasileiras. Também é um dos resíduos mais reciclados no país, e a polpa produzida tem sido utilizada para os mais diversos fins, além da fabricação do próprio papel e do papelão. Dentre eles, pode-se citar a produção de painéis de isolamento, a composição do gesso cartonado, a fabricação de chapa dura e de telhas de fibrocimento. Assim, a reciclagem de papel é uma metodologia que já vem demonstrando uma importância cada vez maior no sistema econômico e na preservação ambiental; o mesmo poderia ocorrer em relação aos resíduos gerados pela agroindústria. Alguns podem ser utilizados com sucesso na confecção de painéis de partículas, com destaque para a casca de amendoim [3].

A cultura do amendoim representa atualmente 150 mil ha plantados no Brasil. A produção de amendoim com casca no Brasil, em 2012, foi de 296 mil t [4].

Em relação à Argentina, o país é atualmente o principal fornecedor de amendoim ao mercado mundial, com 750 mil t/ano. A Argentina exporta 95\% de sua produção e é reconhecida pelos mercados europeus e americanos como um país líder nesta cultura, pela qualidade de seus grãos. Países como Índia e EUA são importantes produtores de amendoim em escala mundial, e também grandes consumidores.

A fração de casca do amendoim representa $30 \%$ da produção do grão e seu principal uso é, atualmente, como combustível para caldeira e alimento para gado. Consideradas um resíduo agroindustrial, as cascas de amendoim disponíveis constituem um recurso abundante e acessível para o desenvolvimento de produtos reciclados.

Uma opção para agregar valor a esses resíduos está na fabricação de painéis de partículas, pois apresentam custo inferior em relação aos painéis de madeira compensada. As propriedades mecânicas desse produto permitem sua aplicação em diversas áreas da construção civil, arquitetura e indústria moveleira.

CARASCHI [5] estudou a viabilidade de produzir painéis à base de casca de amendoim como elemento de reforço e embalagens cartonadas e plásticas como matriz polimérica. Foram avaliadas as propriedades físico-mecânicas do compósito produzido. Os resultados foram analisados segundo a norma ANSI A208.1:1993 [6], que especifica as propriedades de desempenho requeridas para painéis de partículas e que permitiu classificar os painéis como de baixa densidade, sendo indicados para uso em forros, divisórias, revestimento decorativo e demais aplicações que requerem as mesmas propriedades físicas e mecânicas. 
Seguindo a tendência de desenvolvimento de produtos sustentáveis e de utilização de resíduos da agroindústria como proposta para agregar valor a este material, de modo a evitar seu descarte ou mesmo emprego apenas como material destinado à queima, o presente trabalho teve como finalidade desenvolver um estudo para avaliar a viabilidade técnica de fabricação de painéis aglomerados à base de casca de amendoim, por meio da determinação das suas propriedades físico-mecânicas com tratamento superficial preservante e impermeabilizante.

\section{MATERIAIS E MÉTODOS}

Os painéis de partículas à base de casca de amendoim foram fabricados no Laboratório do Instituto de Tecnologia de La Madera (UNSE - Argentina), utilizando uma prensa termohidráulica com aquecimento, capacidade de carga de $100 \mathrm{t}$ e seguindo as recomendações detalhadas por MALONEY [7]. O processo iniciou-se com a coleta do material. Na sequência, os resíduos foram secos e utilizados com dimensões médias inferiores a $10 \mathrm{~mm}$. A Figura 1 apresenta ilustração das cascas de amendoim utilizadas no trabalho, com destaque para a geometria côncava-convexa e densidade real das partículas igual a $0,81 \mathrm{~g} \mathrm{~cm}^{-3}$.

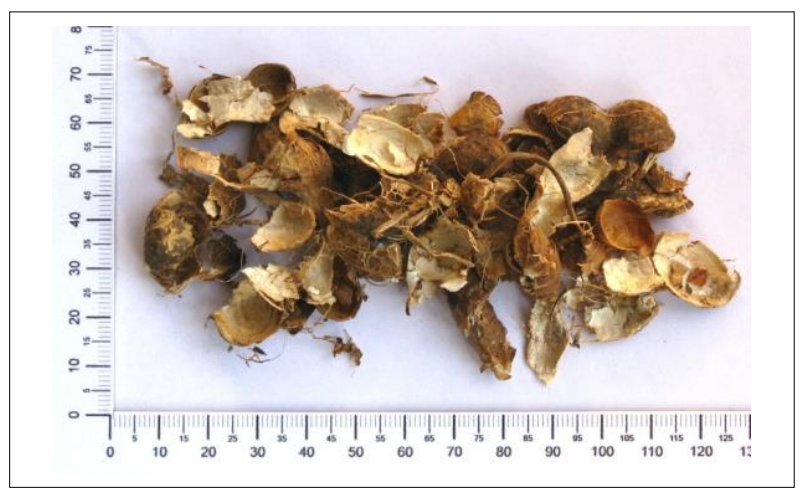

Figura1: Cascas de amendoim (escala em mm).

Posteriormente, foi utilizado um misturador planetário para homogeneizar as partículas com a resina ureia-formaldeído (Coladur 65\%) fornecida pela empresa Jucarbe S.A. - Argentina, com conteúdo de sólidos mínimos de $65 \%$, densidade de $1,26 \mathrm{~g} \mathrm{~cm}^{-3}$, tempo de formação de gel a $100{ }^{\circ} \mathrm{C}$ de 6 min e viscosidade a 25 ${ }^{\circ} \mathrm{C}$ de 950 cps. Foi utilizada uma quantidade de resina ureia-formaldeído de $10 \%$ em relação à massa de partículas de cascas de amendoim. Foi utilizado, também, catalisador para moldagem a quente (Coladur c28) produzido pela empresa Jurcabe S.A. - Argentina, na proporção de 5\% da massa da resina.

Após a mistura, o material foi colocado em um molde formador de colchão e encaminhado à prensa termohidráulica. Para a confecção dos painéis, foram utilizados $25 \mathrm{MPa}$ de pressão e temperatura de $100{ }^{\circ} \mathrm{C}$.

Foram confeccionados painéis de partículas com dimensões nominais de 400 x 400 x $11 \mathrm{~mm}$ e densidade média de $0,70 \mathrm{~g} \mathrm{~cm}^{-3}$. Desses painéis, foram retirados aleatoriamente 12 corpos de prova para cada ensaio físico-mecânico e para cada tratamento avaliado.

Após a cura dos painéis, foram selecionados 12 corpos de prova para serem avaliados sem tratamento preservativo (T1-M), 12 corpos de prova com pintura superficial impermeabilizante de resina poliuretana bicomponente à base de óleo de mamona [PU de mamona] (T2-M) e 12 corpos de prova com pintura superficial preservante de fungicida e inseticida para madeira da marca Tersuave, elaborado à base de Cipermetrina e TCMTB (T3-M), com o fim específico de observar os efeitos destes produtos na preservação do painel e nas propriedades físico-mecânicas do compósito, quando comparado com painéis de partículas sem tratamento preservativo (T1-M).

Os ensaios de caracterização físico-mecânica (densidade, absorção de água [AA], inchamento em espessura [IE], módulo de ruptura [MOR] e módulo de elasticidade [MOE]) foram realizados segundo as prescrições do documento normativo ASTM D1037:2006 [8]. A caracterização físico-mecânica foi realizada aos 9 meses de idade, após armazenamento em condições interiores de atmosfera controlada, permitindo assim a observação das condições de durabilidade proporcionadas pelo tratamento preservativo à base de fungicida e inseticida.

A análise estatística foi realizada considerando-se um delineamento experimental inteiramente casualizado. Para avaliar o efeito dos tratamentos (Tabela 1), foi utilizada a análise de variância para todos os 
ensaios. As análises foram realizadas ao nível de $95 \%$ de probabilidade, tanto para o teste $\mathrm{F}$ como para o teste de Tukey, e foi possível comparar resultados de propriedades físico-mecânicas de painéis sem tratamento (T1-M) com aqueles de painéis tratados com fungicida e inseticida (T3-M).

Tabela 1: Tratamentos avaliados.

\begin{tabular}{l|l}
\hline PAINEL & TRATAMENTO \\
\hline T1-M & Sem tratamento \\
\hline T2-M & PU Mamona \\
\hline T3-M & Fungicida + Inseticida \\
\hline
\end{tabular}

\section{RESULTADOS}

Este item apresenta resultados da caracterização visual e físico-mecânica dos painéis de partículas à base de casca de amendoim e resina ureia-formaldeído (T1-M, T2-M e T3-M). São apresentados valores médios de densidade, inchamento em espessura (IE), absorção de água (AA), módulo de ruptura (MOR) e módulo de elasticidade (MOE).

\subsection{Aspecto visual}

Os painéis confeccionados com cascas de amendoim apresentaram um aspecto atrativo de aparência e textura em relação aos seus similares de partículas de madeira. Caracterizaram-se por uma variedade de tons ocres, cujo aspecto natural pode estimular seu uso na arquitetura de interiores (Figura 2).

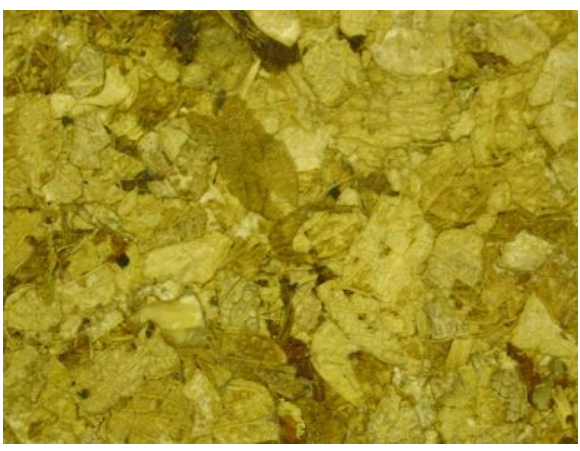

Superfície do painel (T1-M)

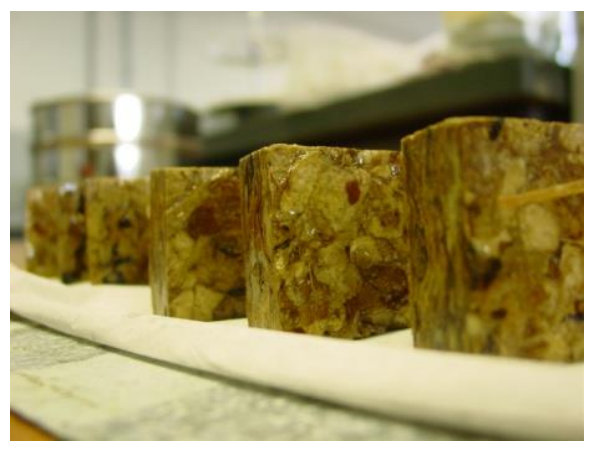

Painel com revestimento PU de mamona (T2-M)

Figura 2: Vista da superfície dos painéis de partículas de casca de amendoim

A Figura 3 apresenta uma vista lateral dos painéis aos nove meses de idade. Os painéis T1-M apresentaram degradação biológica ocasionada por insetos após o período de exposição em ambiente coberto e atmosfera controlada.

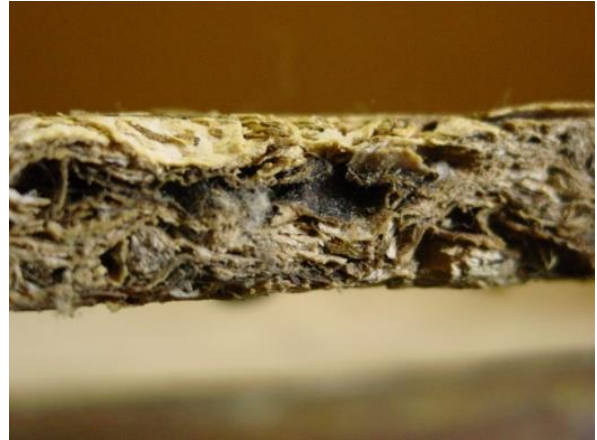

Sem tratamento preservante (T1-M)

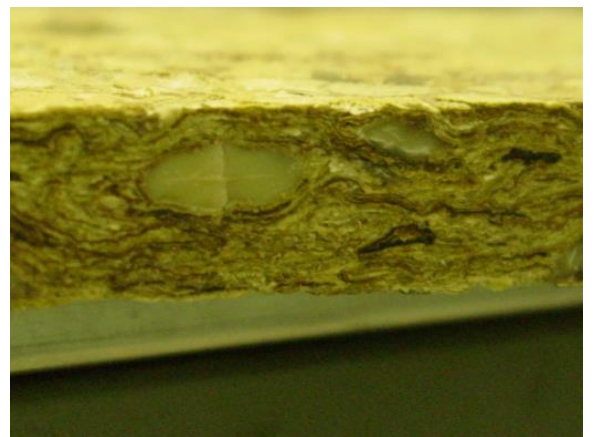

Com tratamento preservante (T3-M) 

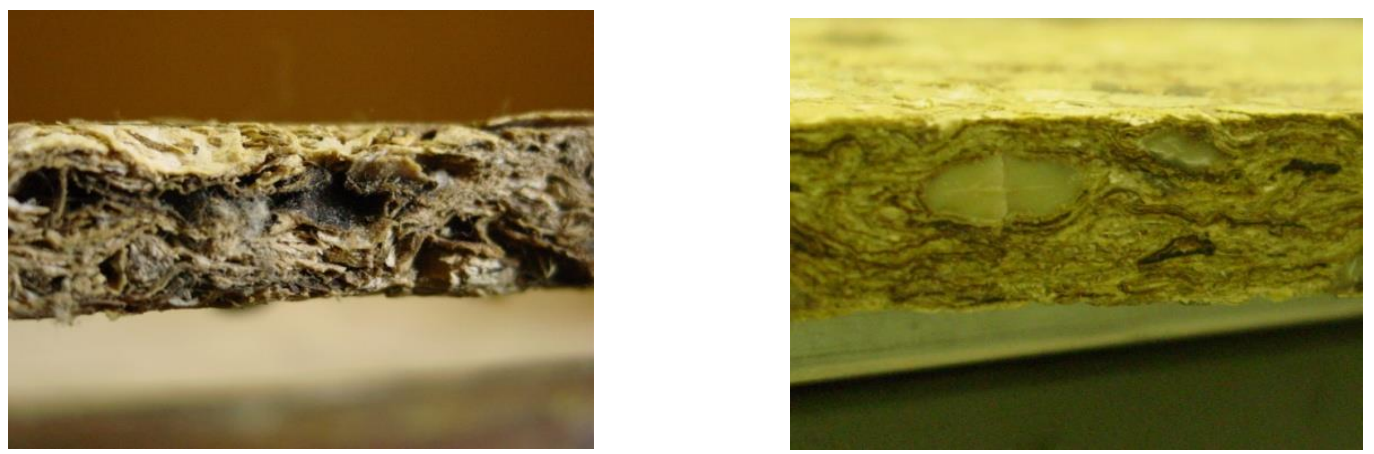

Figura 3: Vista da superfície lateral dos painéis de casca de amendoim.

\subsection{Propriedades físico-mecânicas}

A Tabela 2 apresenta valores médios de propriedades físico-mecânicas dos painéis de casca de amendoim T1-M e T3-M. São indicados também resultados da análise estatística inferencial (teste de Tukey a 5\% de significância).

Tabela 2: Valores médios de propriedades físico-mecânicas dos painéis de casca de amendoim

\begin{tabular}{l|l|l|l|l|l}
\hline $\begin{array}{l}\text { PAINEL DE CASCA DE AMENDOIM + } \\
\text { RESINA UREIA-FORMALDEÍDO }\end{array}$ & $\begin{array}{l}\text { DENSIDADE } \\
\left(\mathrm{g} / \mathrm{cm}^{3}\right)\end{array}$ & $\begin{array}{l}\text { IE 24 h } \\
(\%)\end{array}$ & $\begin{array}{l}\text { AA 24 h } \\
(\%)\end{array}$ & $\begin{array}{l}\text { MOR } \\
(\mathrm{MPa})\end{array}$ & $\begin{array}{l}\text { MOE } \\
(\mathrm{MPa})\end{array}$ \\
\hline $\mathrm{T} 1-\mathrm{M}$ & $0,69^{\mathrm{a}}$ & $16^{\mathrm{a}}$ & $70^{\mathrm{a}}$ & $4,37^{\mathrm{a}}$ & $590^{\mathrm{a}}$ \\
\hline $\mathrm{T} 3-\mathrm{M}$ & $0,83^{\mathrm{b}}$ & $16^{\mathrm{a}}$ & $47^{\mathrm{b}}$ & $5,34^{\mathrm{b}}$ & $762^{\mathrm{b}}$ \\
\hline
\end{tabular}

*Médias seguidas pela mesma letra, na mesma coluna, não diferem entre si pelo teste de Tukey ao nível de 5\% de significância.

A Figura 4 apresenta a relação entre tensão por deformação para corpos de prova sem tratamento preservativo (T1-M) e com tratamento preservativo (T3-M) aplicado sobre a superfície dos painéis de cascas de amendoim. Nota-se uma redução na resistência do painel T1-M, ocasionada pela degradação das partículas por insetos. 


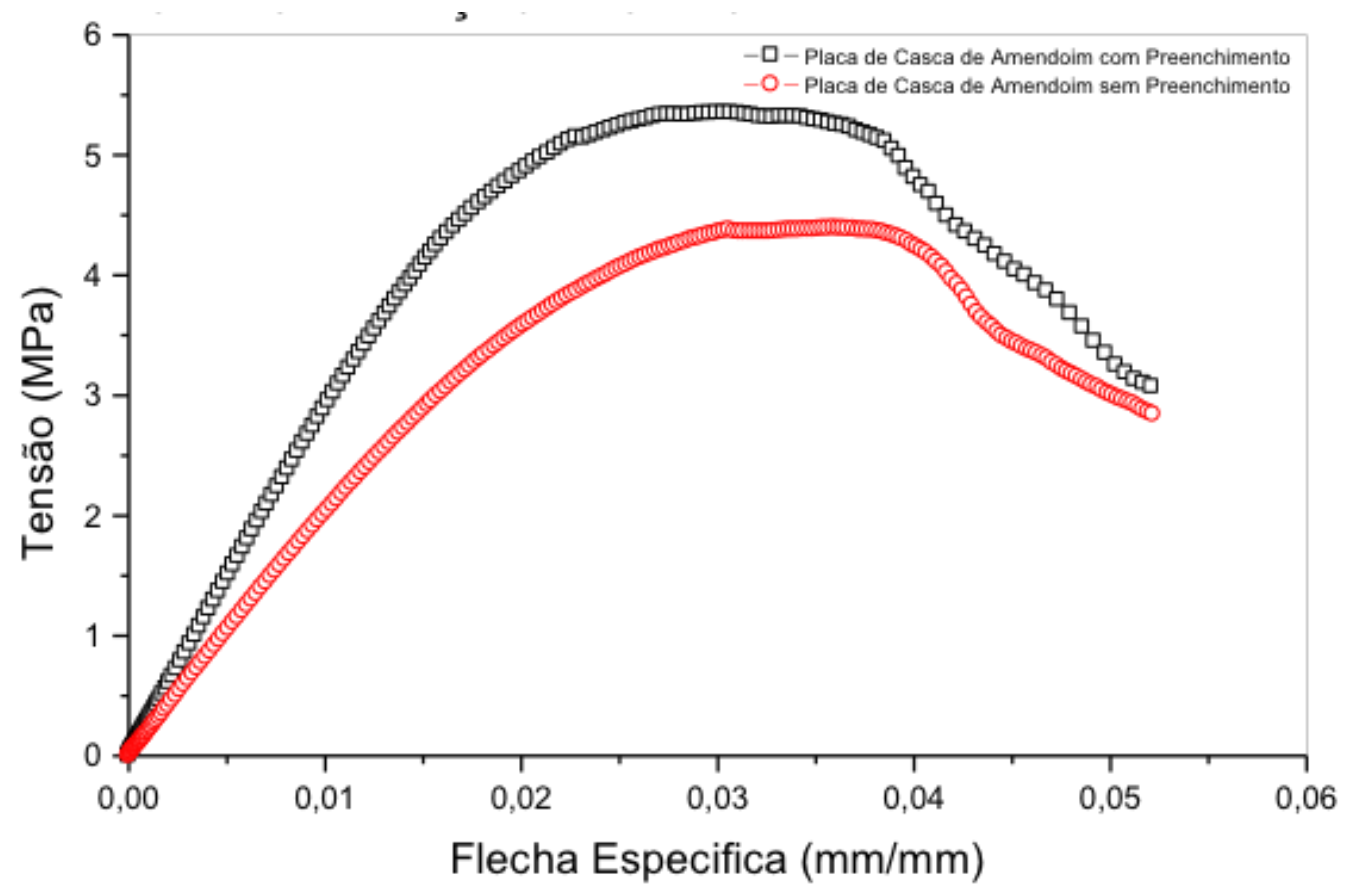

Figura 4. Diagrama tensão x deformação (T1-M e T3-M)

A Figura 5 apresenta valores de IE e AA dos corpos de prova de painéis de casca de amendoim (T1-M, T2-M e T3-M). Para os painéis T2-M, com revestimento superficial de resina PU mamona, foi observada uma diminuição significativa do IE e da AA e, na maioria dos casos, estas propriedades apresentaram valores nulos. Para os painéis T1-M e T3-M, após 24h de imersão, o IE ficou em torno de $15 \%$ e a AA entre $47 \%$ e $70 \%$.
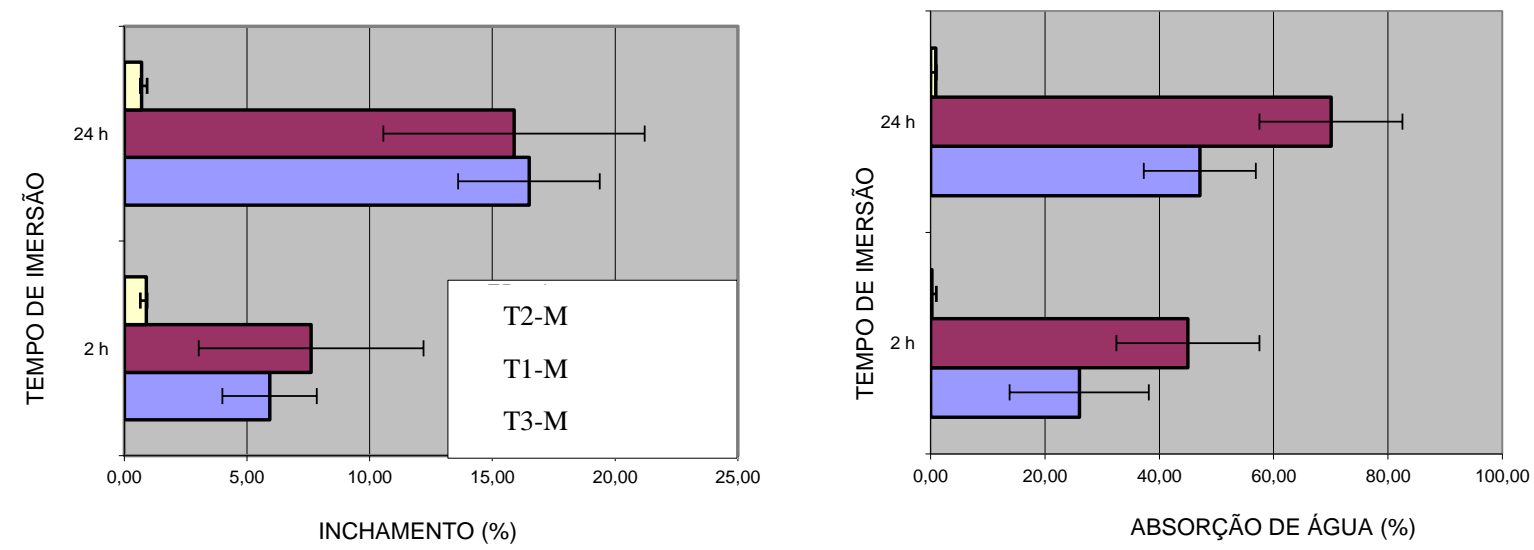

Figura 5. Valores médios: (a) IE e (b) AA.

\section{DISCUSSÃO}

A degradação biológica (Figura 03) observada nos painéis de partículas (T1-M) ocasionou um decréscimo nas propriedades mecânicas, conforme apresentado na Tabela 2. Esses resultados evidenciam a necessidade de utilização de tratamento preservante (fungicida e inseticida) para aumentar a durabilidade desses painéis. Pelas indicações do documento normativo [5], os painéis à base de casca de amendoim e resina ureiaformaldeído, em estudo, podem ser classificados como de média e alta densidade, mas não atendem aos requisitos mínimos de resistência e elasticidade estabelecidos para aplicação estrutural; no entanto, apresentam características físico-mecânicas que permitem sua aplicação como revestimento de superfície em ambientes protegidos de sol e chuva. Essa indicação corrobora os resultados obtidos por CARASCHI [6], que estudou a viabilidade de produzir painéis à base de casca de amendoim como elemento de reforço, e de 
embalagens cartonadas e plásticas como matriz polimérica. Os resultados foram analisados segundo a norma ANSI A208.1:1993 [5], que especifica as propriedades de desempenho requeridas para as chapas de partículas e permite classificar os painéis como de baixa densidade, podendo ser utilizados como forros, divisórias, revestimento decorativo e demais aplicações que requerem propriedades físicas e mecânicas semelhantes.

Os resultados experimentais de IE (24h) dos painéis T1-M, T2-M e T3-M são semelhantes aos apresentados por GULER [9], que estudou a viabilidade da produção de painéis de partículas aglomeradas com partículas de casca de amendoim e casca de pinho negro europeu. $\mathrm{O}$ teste consistiu em produzir painéis com densidade $0,7 \mathrm{~g} \mathrm{~cm}^{-3}$, com $0 \%, 25 \%, 50 \%, 75 \%$ e 100\% de casca de amendoim agregados à mistura, juntamente com o adesivo de ureia-formaldeído. Os resultados obtidos indicam que ocorreu maior absorção de água nos painéis avaliados com 75\% de casca de amendoim, apresentando os registros de 63,3\% e 73,9\% de AA em 2 e 24 h de imersão, e $13,78 \%$ e 19,84\% de IE.

Os resultados de MOR e MOE dos painéis T1-M e T3-M foram superiores aos encontrados por CHAMMA E LEÃO [10], que estudaram a viabilidade de resíduos como a casca do amendoim, casca do arroz e aparas de embalagens plásticas para a produção de painéis e sua aplicação em construções. Nesse trabalho, foram realizados agrupamentos entre esses resíduos, com proporções que resultaram em dezoito tratamentos, todos eles estudados com três repetições. Nos tratamentos realizados, os valores médios de MOE variaram de 79 a $332 \mathrm{MPa}$. Os valores médios do MOR variaram de 0,5 a 3,3 MPa, o que indica que o processo de fabricação exige cuidado e a adoção de pressão controlada e maior proporção de material plástico, garantindo uma maior estabilidade dimensional e melhor adesão entre as partículas, assegurando, desta maneira, melhores propriedades mecânicas para os painéis.

Os painéis com revestimento externo de resina PU de mamona (T2-M) demonstraram bom acabamento estético, baixa AA e, consequentemente, baixo IE (inferior a 5\%), demonstrando a eficiência desse produto como impermeabilizante.

Pelos resultados obtidos, identifica-se a necessidade de desenvolvimento de novos estudos, com o objetivo de intervir nas etapas de processamento dos painéis para garantir aumento nas propriedades mecânicas e possibilitar novos usos para o material. Incorporar partículas de madeira aos painéis de casca de amendoim, aumentar a quantidade de resina, incorporar parafina na mistura e variar o valor da pressão são parâmetros que, se trabalhados, poderão contribuir de modo significativo com o aumento das propriedades mecânicas do material e, consequentemente, proporcionar novos usos para o produto.

\section{CONCLUSÕES}

Com base nos resultados apresentados, é possível tecer as seguintes conclusões:

- A utilização de tratamento preservativo (fungicida e inseticida) é necessária para garantir a integridade do material e, consequentemente, aumentar sua vida útil;

- A resina PU de mamona mostrou-se eficiente na atuação como produto impermeabilizante de painéis de partículas de casca de amendoim;

- As propriedades físico-mecânicas indicam que o material apresenta um potencial de utilização em ambientes internos de residências, construções agrícolas e nos setores moveleiro e decorativo, para aplicação como revestimento de superfícies.

\section{AGRADECIMENTOS}

Os autores agradecem a:

- Centro Experimental de Vivenda Econômica (CEVE) e Conselho Nacional de Investigação Científicas e Tecnológicas (CONICET) e ao Dr. Ricardo Arguello por seu assessoramento;

- Agência Nacional de Promoção Cientifica e Tecnológica (ANPCyT) pelo suporte financeiro ao Projeto de Investigação Científica e Tecnológica (PICT) 33128;

- Ação de Cooperação de Resíduos Agroindustriais: fonte sustentável de materiais de construção do Programa de Ciência e Tecnologia (CyTED);

- Laboratório do Instituto Tecnológico da Madeira da Faculdade de Ciências Florestais, Universidade de Santiago del Estero.

- Fundação de Amparo à Pesquisa do Estado de São Paulo (FAPESP) 


\section{BIBLIOGRAFIA}

[1] NASCIMENTO, M.F., CPH - Chapas de partículas homogêneas - madeiras do nordeste do Brasil, Tese de D.Sc., EESC/USP, São Carlos, SP, Brasil, 2003.

[2] CARASCHI, J.C., LEÃO, A.L., COIADO CHAMMA, P.V., "Produção de painéis a partir de resíduos lignocelulósicos para uso na arquitetura", In: XI Encontro Brasileiro em Madeira e Estruturas de Madeira, CD-Room, Londrina, PR. Jul. 2008.

[3] BRITO, E.O., Sá-ROCHA, J.D., VIDAURRE, G.B., et al., "Propriedades de chapas produzidas com resíduos do fruto de coco e partículas de pinus", Floresta e Ambiente, Seropédica, V.11, n.2, pp. 01- 06, ago.dez. 2004.

[4] COMPANHIA NACIONAL DE ABASTECIMENTO, "Acompanhamento de safra brasileira: grãos, oitavo levantamento", Companhia Nacional de Abastecimento, maio 2012. Brasília, CONAB, 2012.

[5] AMERICAN NATIONAL STANDARDS INSTITUTE, "Mat-formed wood particleboard: Specification", National Particleboard Association, Gaithersburg, ANSI A208, 1-1993.

[6] CARASCHI, J.C., LEÃO, A.L., CHAMMA, P.V.C., "Painéis produzidos a partir de resíduos sólidos para aplicação na arquitetura", Polímeros: Ciência e Tecnologia, São Carlos, SP. v. 19, n. 1, pp. 47-53, 2009.

[7] MALONEY, T.M. "The family of wood composite materials", Forest Products Journal, v. 46, n. 2, pp. 19-26, 1996.

[8] AMERICAN SOCIETY FOR TESTING AND MATERIALS, "Standard test methods for evaluating properties of wood-base fiber and particle panel materials", ASTM D1037-06, Philladelphia, 2006.

[9] GULER, C., COPUR, Y., TASCIOGLU, C., "The manufacture of particleboards using a mixture of peanut hull (Arachis hypoqaea L.) and European Black pine (Pinus nigra) wood chips", Bioresource Technology, v. 99, pp. 2893-2897, 2008.

[10] CHAMMA, P.V.C., LEÃO, A.L., "Aproveitamento de resíduos sólidos na produção de painéis para aplicações arquitetônicas”, Revista Energia na Agricultura, v. 23, n. 2, pp. 73-87, 2008. 